\author{
Pathophysiology \\ of Haemostasis \\ and Thrombosis
}

Pathophysiol Haemost Thromb 2002;32:16-24

Received: J uly 27, 2001

Accepted in revised form: November 26, 2001

\title{
Effects of Aspirin and Clopidogrel versus Oral Anticoagulation on Platelet Function and on Coagulation in Patients with Nonvalvular Atrial Fibrillation (CLAFIB)
}

\author{
Iris Müllera Steffen Massberga Wolfgang Zierhutc Christiane Binz ${ }^{c}$ \\ Alexander Schuster ${ }^{d}$ Silja Rüdiger-von Hoch ${ }^{c}$ Siegmund Braun ${ }^{b}$ \\ Meinrad Gawaz ${ }^{a}$ \\ a1. Medizinische Klinik und Poliklinik, Klinikum rechts der Isar und Deutsches Herzzentrum, \\ bInstitut für Laboratoriumsmedizin, Deutsches Herzzentrum, Technische Universität München, \\ 'Bristol-Myers Squibb, Munich, and dSanofi-Synthelabo, Berlin, Germany
}

\author{
Key Words \\ Aspirin · Clopidogrel · Atrial fibrillation, nonvalvular . \\ Platelet function - Coagulation
}

\begin{abstract}
The aim of the study was to evaluate the effect of two antithrombotic therapies on platelet function and on coagulation in patients with nonvalvular atrial fibrillation (NVAF). Twenty patients with NVAF were treated with aspirin (300 mg/day) and clopidogrel (75 mg/day) for 2 weeks immediately followed by oral anticoagulation (target international normalized ratio 2.0-3.0). Parameters of platelet function and coagulation were evaluated before antithrombotic therapy, at the end of aspirin plus clopidogrel and during subsequent anticoagulation treatment. Aspirin plus clopidogrel significantly inhibited platelet aggregation, fibrinogen receptor activation and release of $\mathrm{P}$-selectin and prolonged in vitro bleeding time ( $p<0.01$ ). Coagulation parameters (platelet-dependent thrombin generation, antithrombin III, thrombin-antithrombin III complex, prothrombin fragment $1+2$ ) were not significantly affected. During the subsequent oral anticoagulation phase platelet function was not substantially reduced; however, coagulation parameters were
\end{abstract}

significantly inhibited ( $p<0.001$ ). The results indicate that combined antiplatelet therapy is superior to aspirin monotherapy in inhibiting platelet function but does not seem to substantially modulate coagulation cascade in patients with NVAF.

Copyright $\odot 2002$ S. Karger AG, Basel

\section{Introduction}

Nonvalvular atrial fibrillation (NVAF) is an important independent risk factor for ischemic stroke. It has a prevalence of $6 \%$ among those patients older than 65 years and accounts for approximately $10-15 \%$ of all ischemic strokes and nearly a quarter of strokes for those older than 80 years [1]. Among those patients with NVAF, the stroke rate is approximately 5 times higher compared to control individuals with sinus rhythm [1].

Antithrombotic therapy has unequivocal efficacy for reducing stroke in patients with NVAF [2]. The occurrence of stroke (ischemic and hemorrhagic) is reduced approximately $60 \%$ by adjusted-dose oral anticoagulation compared with no treatment, about $20 \%$ by aspirin compared with no treatment, and about $40 \%$ by oral anticoagulation compared with aspirin $[1,2]$.

\begin{tabular}{ll}
\hline KARGER & ( ) 2002 S. Karger AG, Basel \\
Fax +4161306 1234 & 1424-8832/02/0321-0016\$18.50/0 \\
$\begin{array}{l}\text { E-Mail karger@karger.ch } \\
\text { www.karger.com }\end{array}$ & $\begin{array}{l}\text { Accessible online at: } \\
\text { www.karger.com/journals/pht }\end{array}$
\end{tabular}

Meinrad Gawaz, MD

1. Medizinische Klinik und Deutsches Herzzentrum

Technische Universität München, Lazarettstrasse 36

D-80636 München (Germany), Tel. +49 8912184012

Fax+49891218 4003, E-Mail gawaz@dhm.mhn.de 
Oral anticoagulation with coumarin derivatives is the most effective antithrombotic therapy to date in reducing the rate of stroke in NVAF and is superior to aspirin, and thus is part of standard recommendations for the management of patients with atrial fibrillation $[1,2]$. However, the mean duration of follow-up in clinical trials generally averaged between 1 and 2 years, and oral anticoagulation may not be as effective in long-term use because anticoagulant therapy is difficult to sustain in many elderly individuals [3]. Data from physician surveys suggest that not all patients with atrial fibrillation that could be treated with anticoagulation receive this treatment [3]. This may be at least in part due to the inconvenience of oral anticoagulation therapy and the increased risk of bleeding $[1,2]$. Thus, antithrombotic therapy with aspirin is often used in these patients although less clinical efficacy of aspirin monotherapy has been shown in clinical trials [4].

Besides initiation of primary hemostasis platelets play a central role in the regulation of coagulation [5]. The activated platelet surface promotes prothrombin activation, and therefore thrombin generation by providing a catalytic surface for the assembling of plasma factors of the prothrombinase complex [5]. Antiplatelet therapy with GPIIb-IIIa antagonists has been shown to reduce plateletdependent thrombin generation and thus inhibits coagulation [6]. Recently, combined antiplatelet therapy in patients with coronary artery disease undergoing stent implantation has improved the clinical outcome significantly [7]. Moreover, the use of aspirin and thienopyridines (clopidogrel or ticlopidine) together has shown synergistic antiplatelet effects [8].

The purpose of the present sequential treatment study was to evaluate the synergistic effect of aspirin and clopidogrel on platelet function, platelet-dependent thrombin generation and coagulation parameters in patients with NVAF and to compare the results with oral anticoagulation therapy.

\section{Methods}

Monoclonal Antibodies and Reagents

Monoclonal antibody (mAb) anti-CD42 (anti-GPIb, clone SZ 2) was obtained as phycoerythrin conjugate (Immunotech, Marseille, France). Anti-CD41 (anti-GPIIb-IIIa, clone 84H10, Immunotech) is a complex-specific mAb that recognizes surface expression of glycoprotein IIb-IIIa. mAb anti-PAC-1 (Becton Dickinson, Heidelberg, Germany) is directed against the activated platelet fibrinogen receptor [9]. Anti-CD62P (clone CLBThromb/6) is directed at P-selectin present on the surface of degranulated platelets (Immunotech). Plasma levels of $\beta$-thromboglobulin ( $\beta$-TG) and platelet factor 4 (PF4) were evaluated with commercially available ELISA kits (Assera- chrom Roche Diagnostics). D-dimers were determined by an automated micro-latex-based homogenous immunoassay (Tina-quant, Roche Diagnostics). Protein $\mathrm{C}$ and $\mathrm{S}$ were measured with an automated function clotting test (Instrumentation Laboratory). Reagents for the determination of prothrombin fragment $1+2(\mathrm{~F} 1+2)$ and the thrombin-antithrombin III complex (TAT) by ELISA and for the chromogenic determination of antithrombin III (AT III) were from Dade Behring. Collagen/ADP- or collagen/epinephrine-stimulated in vitro bleeding time (PFA occlusion time) was determined by the platelet function analyzer PFA-100 from Dade Behring.

\section{Patients and Study Design}

In a prospective, multicenter, open sequential drug treatment study, 20 patients with NVAF were enrolled. None of the patients received any antithrombotic therapy before entering the study. Exclusion criteria were basically identical with the SPAF II trial [1, 2]. After having given written informed consent and exclusion of an atrial thrombus by transesophageal echocardiography, patients were treated with aspirin $(300 \mathrm{mg} /$ day $)$ and clopidogrel (300 mg loading dose followed by $75 \mathrm{mg} /$ day) for 2 weeks. Thereafter, antithrombotic therapy was immediately switched to oral anticoagulation to obtain a target international normalized ratio (INR) of 2.0-3.0. To assess the synergistic effect of clopidogrel with aspirin, platelet function of 20 patients with symptomatic coronary artery disease [all patients were on chronic aspirin $(300 \mathrm{mg} /$ day $)$ treatment for at least 1 month before the study and were scheduled for coronary stent implantation] was evaluated before and $24 \mathrm{~h}$ after administration of clopidogrel (300 $\mathrm{mg}$ loading dose followed by $75 \mathrm{mg} /$ day). The study was performed according to the Declaration of Helsinki and after approval by the Institutional Ethics Committee of the Technische Universität München.

Peripheral venous blood samples were taken with a loose tourniquet to avoid artifacts through a short venous catheter inserted into a forearm vein before the first intake of study drugs, 2 weeks after first administration of aspirin and clopidogrel, and then after further 2-3 weeks when therapeutic anticoagulation with phenprocoumon was achieved (target INR 2.0-3.0). A multiple-syringe sampling technique was used and the first $2 \mathrm{ml}$ of blood were discarded. For ex vivo platelet analysis and coagulation parameters, blood was collected in 3.8\% citrate for platelet aggregation and in CPDA for flow cytometry and analysis of platelet-dependent thrombin generation. Blood for $\beta-T G$ and PF4 analysis was collected into tubes containing citrate, theophylline, adenosine and dipyridamole (TAD tubes BD). All samples were processed immediately after blood drawing.

\section{Platelet Aggregation and Luminometry}

Platelet aggregation ex vivo or in vitro was evaluated by optical aggregometry in citrated blood samples at $37^{\circ} \mathrm{C}$ using a two-channel Chronolog aggregometer (Nobis, Germany) as described [10]. Platelet-rich plasma (PRP) was prepared from citrated whole blood by centrifugation (200 g for $20 \mathrm{~min}$ ). The final platelet count was adjusted to $2 \times 10^{8}$ platelets $/ \mathrm{ml}$ with autologous plasma. $5 \mu \mathrm{l}$ of ADP (final concentration 5 and $20 \mu \mathrm{mol} / 1$, respectively) or TRAP (25 $\mu \mathrm{mol} / \mathrm{l})$ were added to induce platelet activation and aggregation was recorded for $5 \mathrm{~min}$. Maximal aggregation at $5 \mathrm{~min}$ was used as the measurement of aggregation. Simultaneously, release of ATP was determined using the firefly luminometer method [10]. Using a standard dose of added ATP, release of ATP was quantified as nmol/l as described [11]. 
Table 1. Demographic characteristics of study patients with nonvalvular atrial fibrillation $(\mathrm{n}=20)$

\begin{tabular}{lc}
\hline Gender, male/female & $18 / 2$ \\
Age, years & $62.9 \pm 9.3$ \\
Medical history & 2 \\
$\quad$ Ischemic heart disease & 11 \\
Hypertension & 1 \\
$\quad$ Previous TIA (>6 months) & 1 \\
$\quad$ Previous hyperthyroidism & 6 \\
$\quad$ No obvious origin & \\
Echocardiogram & $48 \pm 8$ \\
$\quad$ Diameter of left atrium, mm & 6 \\
Moderate LV impairment & 8 \\
LV hypertrophy & 6 \\
Normal & 2 \\
$\quad$ No obvious origin & 11 \\
Medication & 4 \\
$\quad \beta$-blockers & 7 \\
Digoxin & 3 \\
ACE-inhibitors & 5 \\
Calcium antagonists & \\
Diuretics &
\end{tabular}

Figures represent number unless otherwise indicated. $L V=$ Left ventricle.

\section{Platelet Flow Cytometry}

Ex vivo evaluation of platelets and two-color immunolabeling of platelets were performed in whole blood as described [9]. In brief, $5 \mu \mathrm{l}$ of CPDA-anticoagulated whole blood were added to polypropylene tubes preloaded with $45 \mu \mathrm{l}$ of modified Tyrode's buffer containing saturating concentrations of fluorochrome-conjugated antibodies and platelet agonist ADP (5 and $20 \mu M)$ or TRAP $(25 \mu M)$ as indicated. Platelets were simultaneously stained with phycoerythrin-conjugated anti-CD42b and fluorescein (FITC)-conjugated anti-CD62P, anti-PAC-1 or anti-CD41. After labeling, fixed samples were immediately analyzed within $2 \mathrm{~h}$ by flow cytometry. The mean intensity of immunofluorescence was used as an index of antibody binding and thus of receptor surface expression.

\section{Platelet-Dependent Thrombin Generation}

The assay for thrombin generation was performed as described $[11,12]$. In brief, $500 \mu 1$ of PRP with an adjusted platelet count $(2 \times$ $10^{8} / \mathrm{ml}$ ) were placed in a polypropylene tube and $20 \mu \mathrm{l}$ of $1 \mathrm{~mol} / \mathrm{l}$ $\mathrm{CaCl}_{2}$ were added to start clotting in the absence or presence of $0.2 \mathrm{U} / \mathrm{ml} \alpha$-thrombin or tissue factor. The final concentration of tissue factor was one tenth of that required to clot platelet-poor plasma in approximately $80 \mathrm{~s}$ [11]. At the time intervals indicated, $10 \mu \mathrm{l}$ of the sample were transferred into wells of a microtiter plate containing $90 \mu \mathrm{l}$ of $3.8 \%$ sodium citrate. At the end of the incubation time, $50 \mu \mathrm{l}$ of the $0.5 \mathrm{mmol} / 1$ thrombin substrate $\mathrm{S}-2238$ (Sigma) in $1 \mathrm{~mol} / \mathrm{l}$ Tris, $\mathrm{pH} 8.1$ were added and the plate was read kinetically for $60 \mathrm{~min}$ at a wavelength of $405 \mathrm{~nm}$ with a reference wave length of $630 \mathrm{~nm}$ on a Dynatech MR5000 reader. The assay was calibrated by testing known amounts of purified human thrombin in each assay. One unit of thrombin per well yields a value of $25 \mathrm{mOD}$ at $60 \mathrm{~min}$. All measurements were performed as triplicate.

\section{Coagulation Parameters}

Prothrombin time, activated prothromboplastin time (aPTT), fibrinogen and AT III were evaluated in citrated blood samples in our routine clinical chemistry department. Concentrations of Ddimers, protein $\mathrm{C}$ and $\mathrm{S}$, prothrombin $\mathrm{F} 1+2$ and TAT were determined in batches according to the manufacturers' description in aliquots of citrated plasma stored at $-70^{\circ} \mathrm{C}$.

\section{Statistical Analysis}

Results are reported as mean \pm standard deviation (SD). Intraindividual differences of ex vivo values were evaluated by $t$ tests and in case of deviations from normal distribution by Wilcoxon signed rank test. A value of $p<0.05$ was regarded as significant.

\section{Results}

\section{Patients}

The clinical characteristics of the 20 patients with NVAF included in the study are summarized in table 1. None of the patients showed any clinical symptoms of stroke or transient ischemic attacks (TIA) during the study observation period. No serious bleeding events occurred during the observation period.

\section{In vitro Bleeding Time, Platelet Aggregation, and Activation of Platelet Fibrinogen Receptor}

To evaluate the effect of combined antiplatelet (aspirin plus clopidogrel) or anticoagulation therapy on platelet function ex vivo, peripheral venous blood samples were drawn before antithrombotic drug administration, 2 weeks after the first administration of aspirin/clopidogrel, and during subsequent anticoagulation after a stable target INR of 2.0-3.0 was achieved (2-3 weeks after start of anticoagulation). The combination of aspirin and clopidogrel significantly prolonged in vitro bleeding time compared to baseline values (collagen/ADP: $\mathrm{p}<0.008$; collagen/epinephrine: $\mathrm{p}<0.001$ ) (fig. 1 ). In contrast, no change in in vitro bleeding time was found during the anticoagulation period (fig. 1). Similarly, platelet aggregation was significantly inhibited by approximately 70\% (ADP: $\mathrm{p}<$ 0.001 ) and 20\% (TRAP: $\mathrm{p}<0.001$ ), respectively, by aspirin/clopidogrel (fig. 2a), whereas no changes compared to baseline values were found during the anticoagulation phase. Likewise, activation of the platelet fibrinogen receptor was significantly decreased during aspirin/clopidogrel but not during the anticoagulation phase (ADP: $\mathrm{p}<$ 0.001; TRAP: $\mathrm{p}<0.003$ ) (fig. 3a). 
Fig. 1. Effect of aspirin/clopidogrel versus anticoagulation on in vitro bleeding time. In vitro bleeding time was evaluated before (Pre), at the end of aspirin/clopidogrel (ASA/ Clop) therapy and during subsequent anticoagulation therapy in patients with NVAF. Collagen/ADP (Coll/ADP)- or collagen/epinephrine (Coll/Epi)-induced in vitro bleeding time was determined in citrated whole blood using the platelet function analyzer PFA-100. ${ }^{*} \mathrm{p}<0.01$ compared with baseline values.

Fig. 2. Effect of aspirin/clopidogrel (ASA/ Clop) versus anticoagulation on platelet aggregation. Platelet aggregation was determined in PRP before (Pre), at the end of aspirin/clopidogrel therapy and during subsequent anticoagulation therapy in patients with NVAF (a). In addition, the effect of clopidogrel on platelet aggregation was evaluated in patients with coronary artery disease $(\mathrm{CAD}, \mathbf{b})$ that were on chronic aspirin $(300$ $\mathrm{mg} /$ day) treatment before and $24 \mathrm{~h}$ after clopidogrel administration (300 mg loading dose followed by $75 \mathrm{mg} /$ day). Aggregation was induced through addition of ADP (5 or $20 \mu \mathrm{mol} / \mathrm{l})$ or TRAP $(25 \mu \mathrm{mol} / \mathrm{l}) .{ }^{*} \mathrm{p}<0.01$ compared with baseline values.

Fig. 3. Effect of aspirin/clopidogrel (ASA/ Clop) versus anticoagulation on fibrinogen receptor activation. Activation of platelet fibrinogen receptor was determined in blood samples obtained from patients with NVAF (a) and coronary artery disease $(\mathrm{CAD}, \mathbf{b})$ as described above in the legend to figure 2 using an activation-dependent PAC-1 monoclonal antibody. Platelets were left unstimulated or were activated ex vivo with ADP (5 or $20 \mu \mathrm{mol} / \mathrm{l})$ or TRAP $(25 \mu \mathrm{mol} / \mathrm{l}) .{ }^{*} \mathrm{p}<$ 0.01 compared with baseline values.
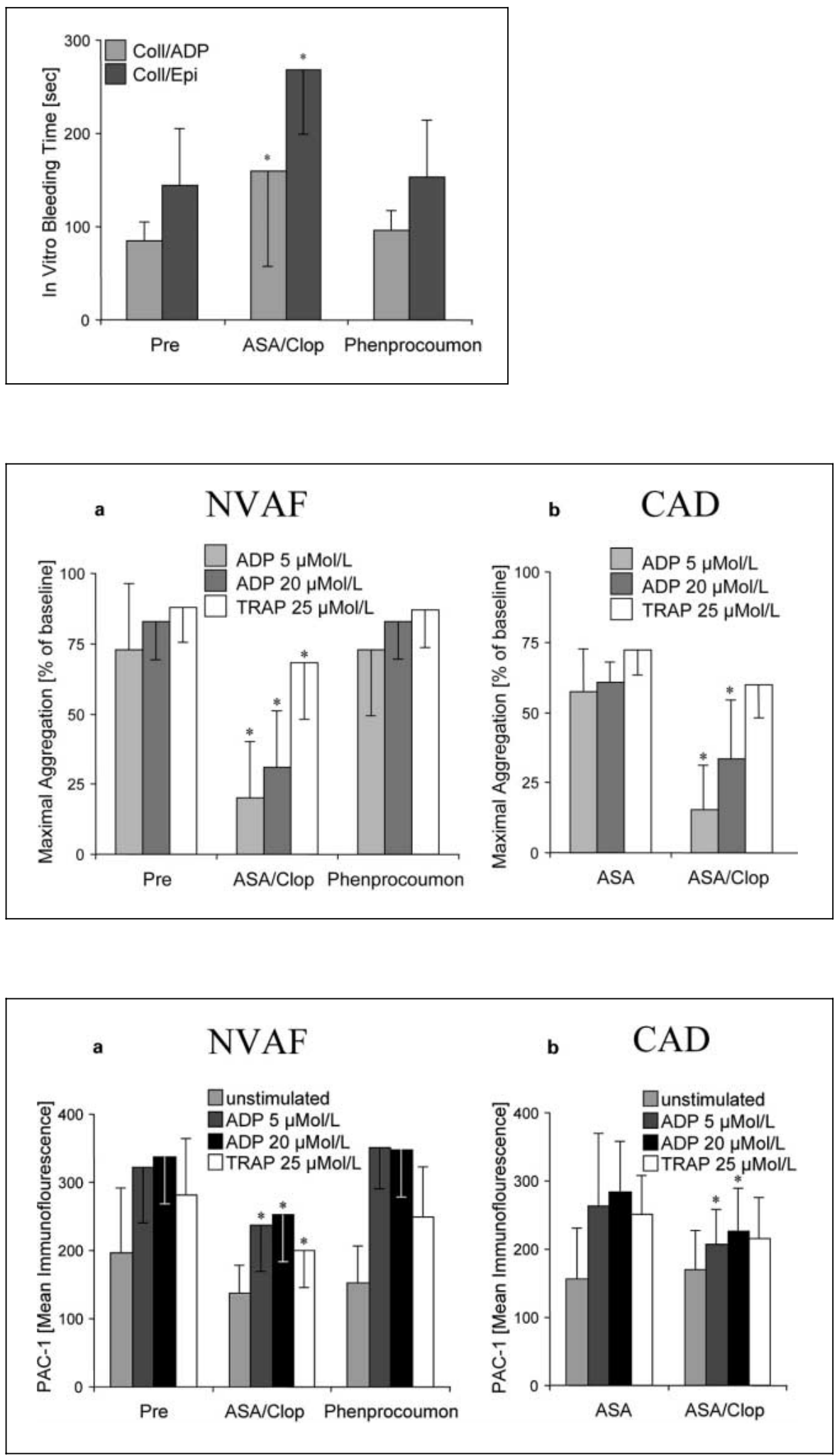
Fig. 4. Effect of aspirin/clopidogrel (ASA/ Clop) versus anticoagulation on $\alpha$-degranulation. Release of $\alpha$-granule determined in blood samples obtained from patients with NVAF (a) and coronary artery disease $(\mathrm{CAD}, \mathbf{b})$ as described above in legend to figure 2 using a monoclonal antibody directed against P-selectin. Platelets were left unstimulated or were activated ex vivo with $\operatorname{ADP}(5$ or $20 \mu \mathrm{mol} / \mathrm{l})$ or TRAP $(25 \mu \mathrm{mol} / \mathrm{l}) . * \mathrm{p}<$ 0.01 (TRAP: $p=0.01$ ) compared with baseline values.

Fig. 5. Effect of aspirin/clopidogrel (ASA/ Clop) versus anticoagulation on release of dense bodies. Release of dense bodies (ATP release) was determined in blood samples obtained from patients with NVAF (a) and coronary artery disease (CAD, b) as described above in legend to figure 2 using luminometry. Platelets were activated ex vivo with ADP (5 or $20 \mu \mathrm{mol} / \mathrm{l})$ or TRAP $(25 \mu \mathrm{mol} / \mathrm{l}) .{ }^{*} \mathrm{p}<0.001$ compared with baseline values.
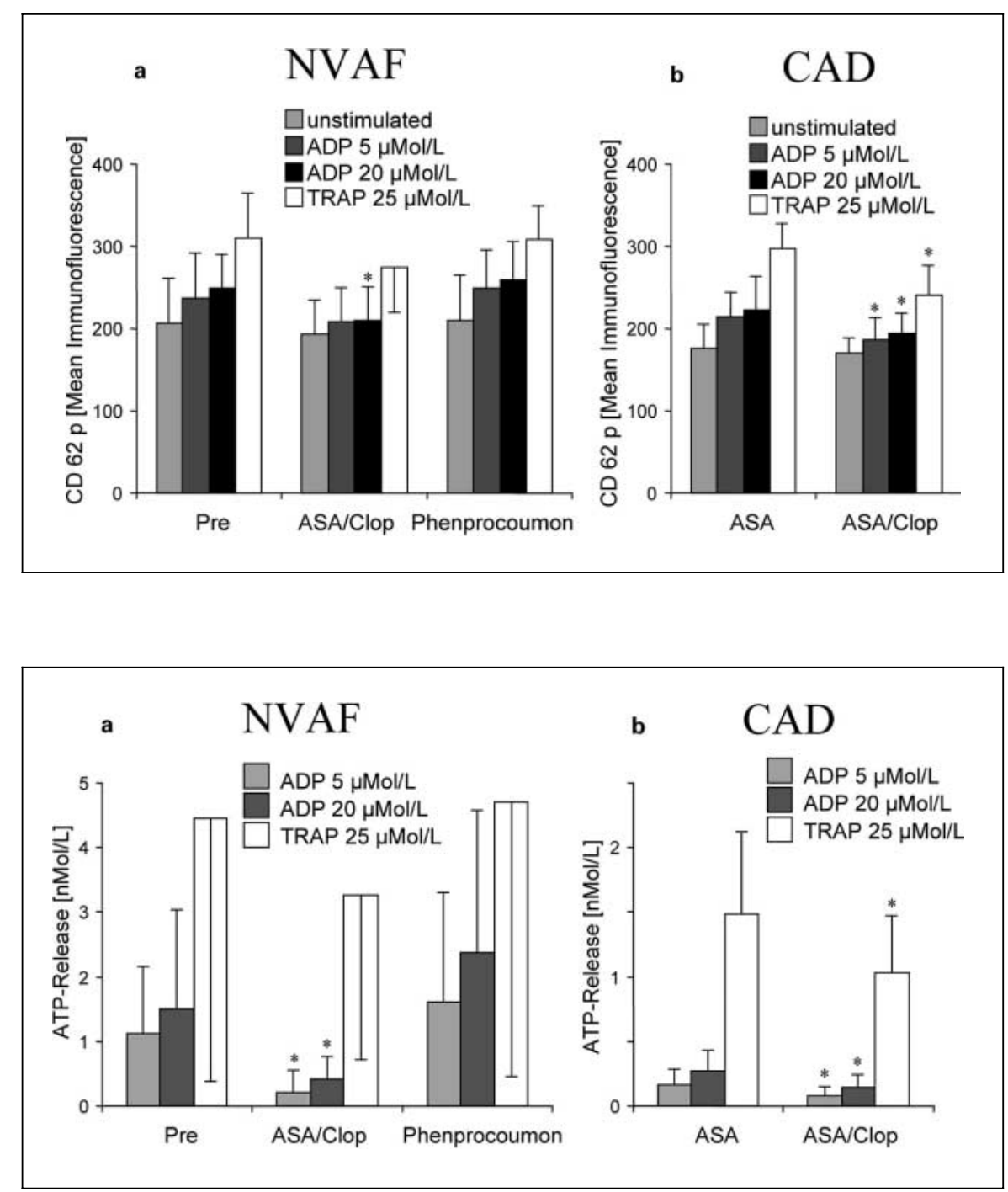

To assess the synergistic contribution of clopidogrel to aspirin with respect to platelet inhibition, platelet function of 20 patients with coronary artery disease that were on chronic aspirin treatment $(300 \mathrm{mg} /$ day $)$ was evaluated before and $24 \mathrm{~h}$ after administration of clopidogrel (300 mg loading dose followed by $75 \mathrm{mg} /$ day). Administration of clopidogrel further inhibited platelet aggregation by approximately $60 \%$ (ADP) or $15 \%$ (TRAP) compared with aspirin monotherapy ( $<<0.01)$ (fig. 2b). Similar results were found for inhibition of platelet fibrinogen receptor $(\mathrm{p}<0.01)$ (fig. $3 b)$.

\section{Platelet Release Reaction}

To evaluate the effect of antithrombotic therapy on the platelet release reaction, surface expression of P-selectin
( $\alpha$-granule release) on ADP ( 5 or $20 \mu \mathrm{mol} / \mathrm{l})$ or TRAP $(25 \mu \mathrm{mol} / 1)$-activated platelets, plasma levels of $\beta$-TG and PF4 (spontaneous in vivo $\alpha$-degranulation), and release of ATP (dense bodies) during aggregation were determined. During aspirin/clopidogrel therapy but not during the anticoagulation period surface expression of P-selectin on activated platelets was significantly inhibited (ADP: $p<$ 0.005; TRAP: $p=0.01$ ) (fig. 4a). Similarly, release of ATP from ADP-stimulated platelets significantly decreased during the aspirin/clopidogrel but not during the anticoagulation phase (fig. 5a) $(\mathrm{p}<0.001)$. No substantial effect of the two tested antithrombotic therapies was found on spontaneous release of $\alpha$-granules as assessed by plasma levels of $\beta$-TG and PF4 (fig. 6). 


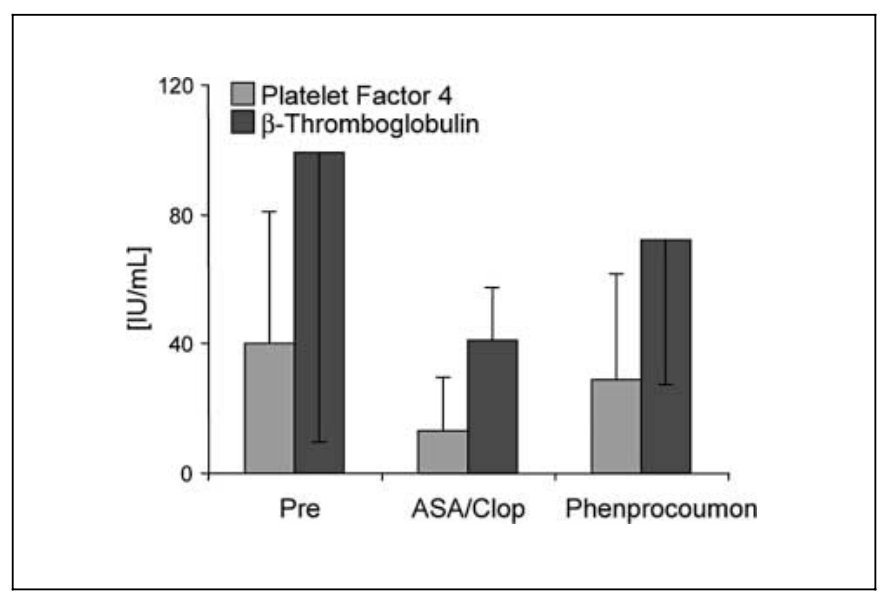

Fig. 6. Effect of aspirin/clopidogrel (ASA/Clop) versus anticoagulation on spontaneous $\alpha$-granule release. Spontaneous release of $\alpha$ granule-specific proteins ( $\beta$-TG, PF4) was determined in plasma samples before (Pre), at the end of aspirin/clopidogrel therapy and during the subsequent anticoagulation therapy in patients with NVAF using luminometry.

Table 2. Platelet-dependent thrombin generation in platelet-rich plasma $(\mathrm{mOD} / \mathrm{min})$

\begin{tabular}{llll}
\hline & Pre & ASA/clopidogrel & $\begin{array}{l}\text { Phenpro- } \\
\text { coumon }\end{array}$ \\
\hline Unstimulated & $20 \pm 13$ & $16 \pm 11$ & $13 \pm 3$ \\
Thrombin & $41 \pm 13$ & $39 \pm 12$ & $16 \pm 5^{*}$ \\
Tissue factor & $52 \pm 13$ & $49 \pm 9$ & $28 \pm 5^{*}$ \\
\hline
\end{tabular}

$* \mathrm{p}<0.001$ compared with baseline values.

Pre $=$ Before therapy; ASA = aspirin.
As described above for aggregation, administration of clopidogrel in patients with coronary disease chronically treated with aspirin significantly reduced both activationinduced P-selectin surface expression $(p<0.01)$ (fig. $4 b)$ and ATP release $(\mathrm{p}<0.001)$ (fig. $5 b)$ when compared with aspirin monotherapy.

\section{Platelet-Dependent Thrombin Generation}

No substantial reduction of platelet-dependent thrombin generation compared to baseline values was found during aspirin/clopidogrel therapy, irrespective of whether thrombin generation was initiated through low-dose thrombin or tissue factor (table 2). During the subsequent anticoagulation phase, however, thrombin- as well as tissue factor-initiated platelet-dependent thrombin generation was reduced substantially by approximately $50 \%$ (p< $0.001)$.

\section{Coagulation Parameters}

To assess the effect of antithrombotic therapy on the coagulation system, various parameters that reflect in vivo thrombin generation $(\mathrm{F} 1+2$, TAT complex $)$ and other coagulation parameters (fibrinogen, AT III, Ddimers, protein $\mathrm{C}$ and $\mathrm{S}$, aPTT, thromboplastin time) were evaluated. Compared to pretreatment values, administration of aspirin/clopidogrel did not substantially inhibit any of the tested coagulation parameters (table 3). As expected, during the anticoagulation phase a significant reduction of in vivo thrombin formation $(\mathrm{F} 1+2$ : $\mathrm{p}<$ 0.001; TAT: $\mathrm{p}<0.003)$, of protein $\mathrm{C}$ and $\mathrm{S}(\mathrm{p}<0.001)$ and an increase of AT III $(\mathrm{p}<0.001)$ was found (table 3$)$. No change of plasma fibrinogen and D-dimers during anticoagulation was noted (table 3 ).

Table 3. Coagulation parameters

\begin{tabular}{lccc}
\hline & Pre & ASA/clopidogrel & Phenprocoumon \\
\hline INR & $1.0 \pm 0.1$ & $1.0 \pm 0.1$ & $2.2 \pm 0.9^{*}$ \\
aPTT, s & $25 \pm 2$ & $25 \pm 2$ & $37 \pm 6^{*}$ \\
Fibrinogen, mg/dl & $315 \pm 79$ & $329 \pm 101$ & $323 \pm 97$ \\
AT III, \% & $91 \pm 14$ & $94 \pm 14$ & $100 \pm 14^{*}$ \\
D-dimers, mg/l & $0.4 \pm 0.2$ & $0.4 \pm 0.2$ & $0.3 \pm 0.1$ \\
Protein C, \% & $118 \pm 32$ & $113 \pm 27$ & $41 \pm 31^{*}$ \\
Protein S, \% & $114 \pm 22$ & $111 \pm 20$ & $70 \pm 16^{*}$ \\
Prothrombin F1 + 2, nmol/l & $0.8 \pm 0.3$ & $0.8 \pm 0.2$ & $0.2 \pm 0.1^{*}$ \\
TAT, $\mu \mathrm{g} / \mathrm{l}$ & $1.1 \pm 0.7$ & $0.9 \pm 0.6$ & $0.7 \pm 0.5^{*}$ \\
\hline
\end{tabular}

$* \mathrm{p}<0.001$ (TAT: $\mathrm{p}<0.003)$ compared with baseline values.

Pre $=$ Before therapy; ASA $=$ aspirin . 


\section{Discussion}

The findings of the present study show that combined antiplatelet therapy consisting of aspirin and clopidogrel produces a strong inhibition of platelet function in patients with NVAF whereas coagulation parameters including platelet-dependent thrombin generation or in vivo thrombin formation do not seem to be modulated substantially. In contrast, although oral anticoagulation efficiently reduces in vivo thrombin formation and in vitro platelet-dependent thrombin generation in the same patients with NVAF, virtually no effect of anticoagulation treatment on platelet function (in vitro bleeding time, aggregation, release reaction) was seen under the experimental conditions applied. The findings of the present study suggest that combined antiplatelet therapy does not substantially modulate the coagulation system but is superior in inhibiting platelet function compared with aspirin monotherapy. Although it has to be shown in upcoming clinical trials, the present data imply that aspirin and clopidogrel used together is a more potent antithrombotic therapy in patients with NVAF than aspirin monotherapy. Therefore combined antiplatelet therapy may be of clinical benefit for NVAF patients in whom anticoagulation therapy cannot be used.

Patients with atrial fibrillation are at high risk of atrial thrombus formation and systemic embolization [1, 2]. Thrombus formation is a complicated process involving a tight interplay between platelets, coagulation factors and fibrinolysis. Several mechanisms may cause enhanced platelet activity and hypercoagulability resulting in thrombus formation in atrial fibrillation: (1) irregular atrial wall motion, which causes abnormal atrial blood flow and injury of the endothelial monolayer, (2) enhanced platelet aggregation in areas of abnormal blood flow (fibrillating atrium) and increased adhesion of activated platelets to the endothelium, (3) enhanced tissue factor activity of injured endothelium and subsequently an increase in thrombin formation that results in further platelet activation and fibrin generation, thus thrombus consolidation, and (4) development of hemoconcentration induced through increased secretion of atrial natriuretic peptide and decreased secretion of vasopressin due to left atrial distension [13].

In the past, several studies have shown that both platelet function and coagulation are altered in patients with atrial fibrillation [13-18]. Platelet activation is enhanced in patients with atrial fibrillation as determined by enhanced plasma levels of platelet-derived $\beta$-TG and PF 4 $[13,17-19]$. In addition, in patients with atrial fibrillation elevated levels of coagulation parameters such as TAT, D-dimers, fibrinogen, prothrombin $\mathrm{F} 1+2$ and fibrinopeptide A have been described [14-18, 20,21] indicating a hypercoagulable state. Thus, the risk of thrombus formation in these patients is increased.

There is abundant evidence derived from five primary and one secondary prevention trial that anticoagulation reduces the incidence of stroke and systemic embolism by $68 \%$ [reviewed in 1,2]. The absolute benefit of anticoagulants, however, varies among patients due to the markedly different inherent risk of stroke among patient subgroups. Two of the primary prevention trials, AFASAK and SPAF, and the secondary prevention trial EAFT evaluated aspirin versus placebo, each using a different aspirin dose (AFASAK 75 mg/day, SPAF 325 mg/day, EAFT 300 $\mathrm{mg} /$ day). These studies demonstrated that aspirin reduced the risk of stroke compared with placebo, but the difference between aspirin and placebo was statistically significant only in SPAF [4]. An intention-to-treat analysis showed that aspirin resulted in a decrease in stroke of $18 \%$ in AFASAK and of $44 \%(\mathrm{p}=0.02)$ in SPAF. When data were combined in a meta-analysis, aspirin was shown to diminish the risk of stroke by $36 \%(p=0.03)$ and the combined rate of stroke, systemic embolism, or death by $28 \%(\mathrm{p}=0.02)$ [4]. Although anticoagulant therapy is superior to aspirin monotherapy, it is often difficult to sustain especially in elderly individuals [3], is associated with a higher risk of severe bleeding complications [1,2] and as a consequence is often withheld in patients with atrial fibrillation. Furthermore, atrial fibrillation is often associated with coronary artery disease where antiplatelet therapy is the standard antithrombotic regime. Thus, although less clinically effective, patients with atrial fibrillation are often treated with aspirin monotherapy instead of anticoagulation.

Recently, aspirin and thienopyridines (clopidogrel or ticlopidine) have been increasingly prescribed together in patients undergoing coronary stent implantation [7] and have been found to be clinically more effective in reducing postinterventional thromboischemic complications than anticoagulation or aspirin monotherapy [22]. Moreover, this regime (aspirin and clopidogrel) seems to be safe and is a well-tolerated therapy [23]. Thus, although mechanisms of thrombus formation might be different in the coronary artery and in the atrium, the question arises of whether dual antiplatelet therapy might be an alternative treatment to aspirin monotherapy or oral anticoagulation in NVAF patients.

The present study demonstrates that aspirin and clopidogrel together is more effective in inhibiting platelet 
function than aspirin monotherapy in patients with NVAF. Because platelet activation triggers thrombus formation and coagulation, this implies that dual antiplatelet therapy might be more effective than aspirin monotherapy in reducing embolic events in patients with NVAF. However, in the present study neither thrombin generation nor the coagulation cascade appears to be substantially modulated by aspirin and clopidogrel. Yet, in another study clopidogrel did reduce thrombin generation triggered by low concentrations of tissue factor in the rat [24]. However, the question arises of whether combined antiplatelet therapy will be of clinical benefit for patients with NVAF and concomitant severe atherosclerotic diseases in whom anticoagulation is not the antithrombotic therapy of choice in secondary prevention of acute coronary syndromes or atherothrombotic stroke. Interestingly, the results of the European Stroke Prevention Study 2 (ESPS2) [25] showed that a combination antiplatelet therapy with aspirin and a slow-release formulation of dipyridamole was significantly more effective than either drug alone (risk reduction, 36\%) in secondary stroke prevention in recent TIA or stroke patients. The Italian SIFA (Studio Italiano Fibrillazione Atriale) study reported that a new antiplatelet agent, indobufen 100-200 mg twice daily, was as effective as adjusted-dose warfarin in preventing stroke in 916 patients with NVAF and a recent cerebrovascular event [26].

In conclusion, the fact that dual antiplatelet therapy is maintaining stent patency and the results of the ESPS2 and SIFA study support the concept of antiplatelet therapy in patients at risk of thromboembolic events. Therefore, it is of great interest to evaluate the efficacy of dual therapy with aspirin and clopidogrel as an effective alternative antithrombotic therapy in NVAF in clinical trials.

\section{Acknowledgments}

We are grateful to Dr. T. Zwingers (Estimate GmbH, Augsburg, Germany) for the excellent help in statistical analysis of the data. The authors also would like to thank Kirsten Langenbrink and Sandra Kerstan for perfect laboratory evaluation.

\section{References}

1 Hart R, Benavente O, McBride R, Pearce LA: Antithrombotic therapy to prevent stroke in patients with atrial fibrillation: A meta-analysis. Ann Intern Med 1999;131:492-501.

2 Risk factors for stroke and efficacy of antithrombotic therapy in atrial fibrillation. Atrial Fibrillation Investigators. Arch Intern Med 1994; 154:1449-1457.

3 White RH, McBurnie MA, Manolio T, Furberg CD, Gardin JM, Kittner SJ, Bovill E, Knepper L: Oral anticoagulation in patients with atrial fibrillation: Adherence with guidelines in an elderly cohort. Am J Med 1999;106:165-171.

4 The efficacy of aspirin in patients with atrial fibrillation. Atrial Fibrillation Investigators. Arch Intern Med 1997;157:1237-1240.

5 Bevers EM, Comfurius P, Zwaal RF: Mechanisms involved in platelet procoagulant response. Adv Exp Med Biol 1993;344:195-207.

6 Reverter JC, Beguin S, Kessels H, Kumar R, Hemker HC, Coller BS: Inhibition of plateletmediated, tissue factor-induced thrombin generation by the mouse/human chimeric 7E3 antibody. J Clin Invest 1996;98:863-874.

7 Schömig A, Neumann FJ, Kastrati A, Schühlen H, Blasini R, Hadamitzky M, Walter H, Zitzmann-Roth EM, Richardt C, Alt E, Schmitt C, Ulm K: A randomized comparison of antiplatelet and anticoagulant therapy after the placement of coronary artery stents. N Engl J Med 1996;334:1084-1089.
8 Müller I, Seyfarth M, Rüdiger S, Wolf B, Pogatsa-Murray G, Schömig A, Gawaz M: Effect of a high loading dose of clopidogrel on platelet function in patients undergoing coronary stent placement. Heart 2001;85:92-93.

9 Gawaz M, Neumann FJ, Schömig A: Evaluation of platelet membrane glycoproteins in coronary artery disease. Consequences for diagnosis and therapy. Circulation 2000;99:e1-e11.

10 Gawaz M, Ruf A, Pogatsa-Murray G, Dickfeld T, Rüdiger S, Taubitz W, Fischer J, Müller I, Meier D, Patscheke H, Schömig A: Incomplete inhibition of platelet aggregation and glycoprotein IIb-IIIa receptor blockade by abciximab: Importance of internal pool of glycoprotein IIbIIIa receptors. Thromb Haemost 2000;83:915922.

11 Dickfeld T, Ruf A, Pogatsa-Murray G, Müller I, Engelmann B, Taubitz W, Fischer J, Meier O, Gawaz M: Differential antiplatelet effects of various glycoprotein IIb-IIIa antagonists. Thromb Res 2001;101:53-64.

12 Aronson DL, Chang P, Kessler CM: Plateletdependent thrombin generation after in vitro fibrinolytic treatment. Circulation 1992;85: 1706-1712.

13 Sohara H, Amitani S, Kurose M, Miyahara K: Atrial fibrillation activates platelets and coagulation in a time-dependent manner: A study in patients with paroxysmal atrial fibrillation. J Am Coll Cardiol 1997;29:106-112.
14 Kumagai K, Fukunami M, Ohmori M, Kitabatake A, Kamada T, Hori N: Increased intra-cardiovascular clotting in patients with chronic atrial fibrillation. J Am Coll Cardiol 1990;16: 377-380.

15 Gustafsson C, Blomback M, Britton M, Hamsten A, Sbensson J: Coagulation factors and the increased risk of stroke in non-valvular atrial fibrillation. Stroke 1990;21:47-51.

16 Asakura H, Hifumi S, Jokaji H, Saito M, Kumabashiri I, Uotani C, Morishita E, Yamazaki M, Shibata K, Mizuhashi K: Prothrombin fragment F1 + 2 and thrombin-antithrombin III complex are useful markers of the hypercoagulable state in atrial fibrillation. Blood Coagul Fibrinolysis 1992;3:469-473.

17 Feinberg WM, Pearce LA, Hart RG, Cushman M, Cornell ES, Lip GY, Bovill EG: Markers of thrombin and platelet activity in patients with atrial fibrillation: Correlation with stroke among 1,531 participants in the stroke prevention in atrial fibrillation III study. Stroke 1999;30:2547-2553

18 Lip GYH, Lip PL, Zarifis J, Watson RDS, Bareford D, Lowe GDO, Beevers G: Fibrin, Ddimers and $\beta$-thromboglobulin as markers of thrombogenesis and platelet activation in atrial fibrillation. Effects of introducing ultra-lowdose warfarin and aspirin. Circulation 1996; 94:425-431. 
19 Pongratz G, Brandt-Pohlmann M, Henneke KH, Pohle C, Zink D, Gehling G, Bachmann $\mathrm{K}$ : Platelet activation in embolic and preembolic status of patients with nonrheumatic atrial fibrillation. Chest 1997;111:929-933.

20 Yamamoto K, Ikeda U, Fukazawa Hshimada $\mathrm{K}$ : Effects of aspirin on status of thrombin generation in atrial fibrillation. Am J Cardiol 1996;77:528-530.

21 Mondillo S, Sabatini L, Agricola E, Ammaturo T, Guerrini F, Barbati R, Pastore M, Finesch D, Nami R: Correlation between left atrial size, prothrombotic state and markers of endothelia dysfunction in patients with lone chronic nonrheumatic atrial fibrillation. Int J Cardiol 2000; 75:227-232.
22 Leon MB, Baim DS, Popma JJ, Gordon PC, Cutlip DE, Ho KK, Giambartolohei A, Diver DJ, Lasorda DM, Williams DO, Pocock SJ, Kuntz RE: A clinical trial comparing three antithrombotic drug regimens after coronaryartery stenting. N Engl J Med 1996;339:16651671.

23 Bertrand ME, Rupprecht HJ, Urban P, Gershlick AH, Investigators FT: Double-blind study of the safety of clopidogrel with and without a loading dose in combination with aspirin compared with ticlopidine in combination with aspirin after coronary stenting: The clopidogrel aspirin stent international cooperative study (CLASSICS). Circulation 2000; 102:624-629.
24 Herault JP, Dol F, Gaich C, Bernat A, Herbert JM: Effect of clopidogrel on thrombin generation in platelet-rich plasma in the rat. Thromb Haemost 1999;81:957-960.

25 Diener HD, Cunha L, Forbes C, Silvenius J, Smets P, Lowenthal A: European Stroke Prevention Study 2. Dipyridamole and acetylsalicylic acid in the secondary prevention of stroke. J Neurol Sci 1996;143:1-13.

26 Morocutti C, Amabile G, Fattapposta F: Indobufen versus warfarin in the secondary prevention of major vascular events in nonrheumatic atrial fibrillation. SIFA (Studio Italiano Fibrillazione Atriale) investigators. Stroke 1997;28:1015-1021. 